\title{
GESTIÓN DEL RIESGO DE DESASTRES EN EL CARIBE COLOMBIANO DESDE LA ÓPTICA DE ORGANISMOS DE SOCORRO Y ADMINISTRACIONES LOCALES: EL CASO DEL SUR DE ATLÁNTICO
}

\author{
José Hernando Ávila-Toscano ${ }^{1}$ \\ Omar Augusto Vivas Cortés ${ }^{2}$ \\ Ana Herrera Flórez ${ }^{\underline{3}}$ \\ Mireya Jiménez Díaz ${ }^{4}$
}

Recibido el 30 de diciembre de 2014, aprobado el 20 de abril de 2015 y

actualizado el octubre 26 de 2015

DOI: 10.17151/luaz.2016.42.7

\section{RESUMEN}

OBJETIVO: Analizar la forma como se aplica la política de gestión del riesgo en el ámbito territorial en el sur de Atlántico (Caribe colombiano), partiendo de la óptica de actores institucionales y de miembros de las comunidades afectadas por desastres invernales que integran organismos de protección civil. MÉTODO: Estudio cualitativo basado en la investigación acción, desarrollado en seis municipios mediante revisión documental, entrevistas y grupos focales. RESULTADOS: reducido nivel de apropiación de la política como herramienta de planeación territorial y desarrollo comunitario por parte de la institucionalidad, baja participación de organismos municipales y escasa integración de la comunidad. CONCLUSIONES: Se resalta la relevancia de los procesos sociales implicados en la aplicación de la política, señalando los desaciertos que los actores en estudio denuncian en la gestión pública.

\section{PALABRAS CLAVE}

Gestión del riesgo, política pública, desarrollo social.

DISASTER RISK MANAGEMENT IN THE COLOMBIAN CARIBBEAN FROM THE PERSPECTIVE OF RELIEF AGENCIES AND LOCAL GOVERNMENT: THE CASE OF THE SOUTHERN AREA OF THE DEPARTMENT OF ATLÁNTICO

\begin{abstract}
OBJECTIVE: To analyze how the risk management policy is applied in the territorial area in the southern part of the department of Atlántico (Colombian Caribbean) starting from the perspective of institutional actors and members of the communities affected by winter disasters who integrate civil protection agencies. METHOD: Qualitative study based on action research, developed in six municipalities through document review, interviews and focus groups. RESULTS: Reduced level of ownership of the policy as a tool for territorial planning and community development by the institutions, low participation of municipal agencies and poor integration of the community. CONCLUSIONS: The relevance of the social processes involved in the implementation of the policy by pointing out the mistakes that the studied actors reported in the carrying out of this process is emphasized.
\end{abstract}

KEY WORDS: Risk management, public policy, social development. 


\section{INTRODUCCIÓN}

El subdesarrollo se presenta como un conjunto de características que limitan seriamente las posibilidades de desarrollo humano, con obstáculos a las iniciativas e inversiones que buscan el incremento de los índices de bienestar, así como la profundización de las cualidades democráticas, situación que además de imposibilitar el goce efectivo de derechos, contiene formas inadecuadas de relacionamiento entre el hombre y sus entornos.

Las condiciones de atraso no son fortuitas, emergen de las prácticas y procesos sociales, económicos y políticos, internacionales y nacionales, que conforman círculos viciosos que alimentan formas inadecuadas de apropiación y uso del territorio y de los recursos (Banco Mundial, 2012). En una mirada Estado-céntrica, se aprecia que estas formas prosperan a pesar de los marcos normativos que los Estados adoptan para salvaguardar y promover el interés general y los intereses colectivos, bien sea con regulaciones para proteger o con políticas de promoción (Llosa y Zodrow, 2011).

Quizá lo único que tenemos claro sobre las probabilidades de sustentar la vida en el planeta es que no estamos de acuerdo en cómo se logrará, los intereses son excluyentes, los conflictos de visiones y las lógicas encontradas se tornan irreconciliables, los imaginarios y las representaciones sociales oscilan entre el cataclismo y la esperanza de una humanidad interplanetaria -propia de la ciencia ficción-. Tensiones que no cambiarán ni se modularán fácilmente.

Esta claridad ha provocado la emergencia y el protagonismo del sistema conceptual que conciben el control de los riesgos de desastres como el asunto central de la vida de las sociedades, de los Estados y de las personas. Ello hace eco de las ideas de teóricos del campo social -como Beck (1998)-, lo que a su vez enmarca la inclinación por afianzar la acción del Estado que se encarga de la regulación y el manejo de los riesgos (Reveiz, 2007; Luján y Echeverría, 2009).

\section{Gestión del riesgo de desastres, su importancia para el desarrollo social}

La gestión del riesgo de desastres (GRD) debe entenderse ante todo como un proceso, ello le define entonces como conjuntos de acciones sistemáticas que tienen la finalidad de identificar, analizar y delimitar la probabilidad de sufrir pérdidas producidas por desastres, lo que permite formular acciones correctivas pertinentes (Keipi, Mora y Bastidas, 2005) y coherentes con la magnitud de los riesgos experimentados. En este sentido, el riesgo es abordado con un enfoque marcadamente preventivo, lo que permite desarrollar acciones que facilitan la mitigación y la preparación, a la vez que también contempla acciones de respuesta y recuperación en los casos en que la experimentación de los desastres es inevitable (Dwyer et al., 2004).

Desde esta concepción, los desastres se materializan como eventos físicos o biológicos que afectan al hombre, su hábitat y sus medios de vida presentes y futuros, por lo cual se trata de fenómenos que superan lo meramente natural o físico, pues contemplan un carácter social y político relevante (García, 2005; Lavell, 2006). Al vincular la cuestión central con el desarrollo por ejemplo, se encuentra que la vulnerabilidad se corresponde con las configuraciones y capacidades de las sociedades y de los gobiernos, de manera que los desastres están en función de decisiones y procesos sociales, económicos y políticos (PNUD, 2013).

El riesgo de desastre se asume entonces como un proceso que requiere de la intervención pública para su manejo, en este sentido, este proceso implica un 
contenido político, lo cual conduce a pensar que el desastre ocurre en la esfera pública, que a su vez está sujeta a decisiones políticas, a concepciones y posturas políticas, a contenidos discursivos, y además, está inmersa en la lógica de la acción pública que considera las políticas públicas como el marco de trabajo. Así pues, aceptar que un evento es un desastre, es una decisión política promovida por actores políticos interesados, decisión que conlleva una mayor inversión y una secuencia de decisiones y acciones en cascada a través del aparato público para el manejo y la recuperación.

La idea de la GRD constituye una forma elaborada de pensar la realidad, que como producto de la modernidad, aporta una lógica que induce la visión prospectiva y preventiva que hace sustentable el desarrollo, superando la orientación al inmediatismo que se marca en los enfoques centrados en la atención y el manejo de los desastres. A largo plazo las miradas centradas en la atención no son viables, dado que un inadecuado asentamiento humano y de las actividades productivas en el territorio, implica una disponibilidad de recursos y capacidades que a la larga ningún Estado se encuentra en condiciones de proveer.

Para los estudiosos, el manejo del riesgo es un requisito sine qua non para el desarrollo (Cardona, 1993; Wiches-Chaux, 1993; Lavell, 2000); en forma paradójica, hasta los más beligerantes y apasionados están de acuerdo en la necesidad de gestionar el riesgo, en ello sí hay convergencias y acuerdos, incluso la posibilidad de que los intereses individuales cedan ante fines superiores es alcanzable; la lógica de los riesgos logra lo imposible por el poder que tiene de comunicar de manera inmediata y con una orientación teleológica la magnitud de los impactos, allí los beneficios particulares se tornan minúsculos al compararles con las afectaciones generales.

La relevancia de la GRD responde a su vez, a un proceso de construcción sociopolítica del territorio, proceso donde se consideran las amenazas y las vulnerabilidades como aspectos determinantes para el uso y apropiación de la tierra, de los recursos y servicios ambientales, y que en forma excluyente posibilita o no la explotación de recursos minerales. Este nivel de construcción se basa en el concierto entre actores de diversos niveles y sectores, entre los cuales se generan mecanismos de participación y capacidad institucional como elementos sustantivos en la gestión del riesgo, de allí el necesario aporte a dicha articulación desde la apuesta política y la administración territorial, dado que carecer de este nivel de articulación menoscaba la posibilidad de ofrecer acciones congruentes con el nivel de exposición a riesgos y aumenta la probabilidad de sufrir daños (Morales-Soto et al., 2008).

\section{GRD en Colombia, el contexto actual}

En Colombia la inclusión de la GRD como una política de desarrollo en la que se asume desde una mirada prospectiva el manejo de las amenazas, constituye una reconfiguración del problema público, de manera que la cuestión central ya no son los desastres sino la armonización de las formas de apropiación y uso del territorio con las posibilidades de un desarrollo sustentable, en un marco de salvaguarda y promoción de derechos ${ }^{5}$.

En la Ley 1523 de 2012 se sustenta precisamente el proceso de GRD como una política de desarrollo para la nación (Congreso de la República, 2012); se trata de una norma trascendental, en tanto reorienta toda la estructura que se venía manejando con la anterior normativa cuyo énfasis radicaba en la atención de desastres (Decreto Ley 919 de mayo 1 de 1989), lo cual se quedó corto ante los numerosos fenómenos naturales y antrópicos que ha venido afrontando el país en 
los últimos años (Lampis, 2010b; Banco Mundial, 2012), por lo tanto era perentorio darle una reorganización al manejo del riesgo.

A partir de esta norma se determina la adopción de la política y la estructura de GRD desde el orden planificado hasta la creación de los estamentos que deben darle sustento a dicha gestión en los tres niveles de gobierno (nacional, departamental y municipal), determinando además la formulación e implementación de planes de gestión del riesgo para priorizar, programar y ejecutar acciones por parte de las entidades del sistema nacional, en el marco de los procesos de conocimiento y reducción del riesgo, y manejo del desastre.

La Ley 1523 de 2012 establece además mecanismos de participación ciudadana e institucionales con el propósito de lograr que la GRD sea una responsabilidad compartida, lo cual es coherente con los aportes teóricos y los antecedentes en la materia, entre los que se resalta el valor de la cooperación multisectorial donde se integre la institucionalidad pública y privada pero también los diversos grupos humanos implicados en el fenómeno (Lavell, 2006, 2007; Thomas, 2011). Asimismo, esta ley estatuye la GRD como una prioridad dentro de la gestión pública, por ello define una serie de instrumentos y organismos de consejo cuya finalidad esencial estriba en la formulación de procesos de conocimiento, información y planificación adecuada del desarrollo local, es decir, la GRD se instaura como una herramienta que contribuye a la planificación y a la gestión pública de la territorialidad (Lavell, 2006; Velásquez, 2011).

Bajo esta visión se esperan inversiones y acciones prospectivas y preventivas, las primeras incluyen la gestión del riesgo en el desarrollo y las segundas forman capacidades de planeamiento del territorio y de dotaciones materiales, así como de formación de competencias para reducir la amenaza, la vulnerabilidad o los impactos.

En resumen, el propósito o espíritu de la Ley 1523 de 2012, es el de conocer, reducir y manejar los riesgos de desastres, mediante la implementación de un sistema orientador y planificador del desarrollo en todos los niveles de gobierno, basado en la gestión ambiental sostenible, la participación comunitaria y en un firme objetivo de generación de conocimiento frente a los procesos de gestión del riesgo para garantizar con ello el bienestar, la seguridad y la calidad de vida de los habitantes de Colombia (Ley 1523 de 2012, Art. 1).

\section{La política pública de GRD en Colombia (Ley 1523 de 2012): su análisis desde el contexto territorial en el Caribe colombiano}

Los apartados precedentes han dejado por sentado el valor que tiene la gestión del riesgo en los procesos de planeación local y en la gobernabilidad general representada en las instituciones del Estado, al respecto, resulta atinente una pregunta puntual en el marco de nuestro estudio: ¿Por qué focalizar el análisis de la política de gestión del riesgo en el Caribe colombiano? Este interrogante tiene una respuesta precisada desde la reiterada situación de emergencias que todos los años viven los municipios de esta zona por las inundaciones y que en muchas ocasiones son de grandes proporciones detonando en desastres como el ocurrido entre entre los años 2010 y 2011, cuando la nación se enfrentó a los efectos devastadores del fenómeno de La Niña, evento conocido en la cotidianidad del país como "la ola invernal". Tales episodios condujeron a la afectación del $7 \%$ de los habitantes del país con un registro de 3'219.239 personas entre damnificados y afectados (BID y Cepal, 2012).

El Caribe colombiano fue una de las regiones con mayor nivel de afectación, y en dicha región especialmente el departamento de Atlántico que se contó como la 
cuarta jurisdicción del país con mayor nivel de pérdidas (Sánchez, 2011), lo cual ha representado una necesaria inversión millonaria que ha superado los $300 \mathrm{mil}$ millones de pesos (Colombia Humanitaria, 2014). En este sentido, la formulación de la Ley 1523 tuvo entre sus antecedentes dichos episodios de desastres, en virtud de que la magnitud de los mismos superó las capacidades estatales y la dimensión de las políticas existentes hasta esa fecha.

Vale aclarar que la magnitud del riesgo para el territorio en mención (y en general podría asumirse que para todo el país) no se restringe exclusivamente a inundaciones, sino que además se trata de jurisdicciones en las cuales existe un alto riesgo de desastres asociados con riesgos naturales y antrópicos que incluyen: a) incendios forestales (quemas incontroladas), b) inundaciones lentas por pérdida de absorción de suelos provocada por la tala indiscriminada de bosques y el uso de fertilizantes con fines agrícolas, c) ganadería extensiva y cegado de ciénagas, d) colmatación y contaminación de cuerpos de aguas por vertimiento de basuras y residuos sólidos, e) erosión y deslizamientos provocados por cauces de arroyos y extracción de materiales para la construcción, f) temporadas de fuertes sequías por déficit hídrico y olas de calor extremo. Además, se presentan eventos de carácter antrópico como incendios y explosiones por almacenamiento y manipulación de combustibles y líquidos inflamables, concentraciones masivas de público en ferias y similares en condiciones de vulnerabilidad por falta de lugares adecuados, según lo reporta un completo informe realizado por la Fundación Red para la Promoción, Prevención, Atención y Recuperación de Emergencias (REDePARES, 2013).

El citado informe señala además debilidades en los procesos de integración de la gestión del riesgo con los instrumentos de planeación local tales como el Plan de Desarrollo y los Esquemas de Ordenamiento Territorial, lo que sin duda limita las posibilidades de ejecución político-administrativa frente a los riesgos de desastres, en la medida que carecer de estrategias claras de atención de este componente desde la planeación local puede afectar la autonomía de la gestión, limitando la posibilidad de definir mecanismos normativos y ejecutivos para incorporarla a la planificación del territorio (Narváez, Lavell y Pérez, 2009).

Así pues, sea la amenaza de origen natural o inducida por el hombre, la responsabilidad sobre la forma en la que se asumen los riesgos es algo que le corresponde tanto a la sociedad como a la institucionalidad política. De hecho, considerar la gestión del riesgo como un instrumento para la consecución del desarrollo, es algo que depende de la importancia que le otorguen las instituciones al tema, y consecuentemente, de su inclusión en la agenda política (PNUD, 2013).

No obstante, si bien son las instancias estatales las que deben marcar el derrotero e instituir la relevancia de la gestión de los riesgos, las comunidades también deben mostrarse activas y comprender que hacen parte de la solución al problema, pues finalmente, son estas quienes son vulnerables, y las que expuestas a las amenazas, y ante una serie de condiciones estructurales adversas -de carácter social, político y económico- (Audefroy, 2007; Herzer, 2011) pueden sufrir diverso grado de afectación producto de la experimentación de pérdidas de sus oportunidades de desarrollo y sus capacidades colectivas (Lampis, 2010a). Es allí donde subyace el problema y donde, igualmente, es menester que se efectúen las acciones necesarias para la reducción del riesgo.

Aun así, quizás una de las mayores dificultades esté en la poca aprehensión del problema por parte de la comunidad, justificada en "la inequidad en los procesos sociales y económicos [que] conllevan a la creación de nuevas condiciones de vulnerabilidad y al aumento de la existente" (Banco Mundial, 2012, p. 63), producto de la inapropiada intervención institucional en el ámbito local, arraigada en la 
ausencia de capacidades institucionales para la apropiación del conocimiento normativo que reglamenta la función pública, así como en la voluntad política para gestionar las soluciones; a lo que se añade, la poca difusión y conocimiento del riesgo en las comunidades.

Las investigaciones que develen el comportamiento de las sociedades, del Estado y del sector privado, son primordiales en los procesos de conocimiento del riesgo. Ciertamente, la investigación aquí desarrollada descubre las representaciones que se tienen por parte de conglomerados sociales y políticos en la región especialmente autoridades y organismos de socorro conformados por miembros de las mismas comunidades-, respecto a la prioridad que se le ha otorgado a la gestión del riesgo en el territorio, en lo concerniente a la implementación de la política de gestión del riesgo -Ley 1523 de 2012- y a las percepciones e imaginarios sociales construidos, en torno al papel y a las responsabilidades del Estado con la sociedad.

De esta forma, el presente análisis contribuye tanto a la observación detallada de la implementación de la política hasta el momento, como al conocimiento de las percepciones desde la institucionalidad y la comunidad, frente a las posibles falencias y obstáculos que han incidido en su implementación.

\section{MÉTODO}

\section{Diseño y estructura del estudio}

Los resultados presentados en este trabajo se derivan del estudio titulado "Análisis de la política de gestión del riesgo en comunidades del Caribe colombiano afectadas por desastres invernales: el caso del sur del departamento de Atlántico", desarrollado con la financiación del Departamento Administrativo de Ciencia, Tecnología e Innovación (Colciencias), el cual se estructuró en un marco cualitativo de análisis, con un diseño centrado en la integración de técnicas etnográficas dirigidas a la descripción analítica del fenómeno expuesto, las cuales se complementaron con procedimientos derivados de la investigación-acción participativa (IAP).

Etnográficamente, el estudio se valió del uso de técnicas como la revisión de documentos y evidencias, el desarrollo de entrevistas con grupos y el registro de campo, tal enfoque facilita un análisis detallado del fenómeno de estudio dirigido a su comprensión señalando tanto los ideales como las contradicciones identificados en la realidad estudiada (Balcázar, González, Gurrola \& Moysén, 2005). Mientras que el uso de la IAP por su parte, permitió identificar la opinión, los puntos de vista y las posiciones adoptadas por los actores implicados con base en el criterio de negociación de demanda (Martí, 2000), es decir, la libre discusión entre los actores para conocer puntos de vista que entretejen concepciones colectivas entre grupos no necesariamente homogéneos.

Este trabajo se cumplió en seis municipios del sur de Atlántico (Repelón, Manatí, Candelaria, Campo de la Cruz, Suan y Santa Lucía), en los cuales se establecieron reuniones con sus representantes legales (alcaldes), funcionarios de las administraciones y directivos y representantes de los organismos de socorro (Cuerpo de bomberos, Defensa Civil, Centros sanitarios, y se incluyó a la Policía Nacional), previa socialización del proyecto, sus objetivos y alcances, y tras la firma del consentimiento informado. 
Inicialmente, los alcaldes o sus delegados pertenecientes a los Consejos Municipales de Gestión del Riesgo de Desastres (CMGRD) cumplieron con el desarrollo de un inventario diseñado por el grupo de investigadores en el cual se evaluó la existencia de documentación oficial, reportes, evidencias y todo tipo de documento que soportara el cumplimiento de acciones relacionadas con la GRD desde las exigencias de la Ley 1523. Este inventario contaba con 34 preguntas separadas en 6 secciones en las cuales se buscaba recabar información relacionada con procesos como: Cumplimiento de las disposiciones generales de la norma, Información sobre el funcionamiento del CMGRD, Estado de las entidades técnicas y de socorro, Manejo financiero de GRD y Participación sociocomunitaria. En todos los casos se solicitaba evidencia fehaciente relacionada con tales aspectos. En total, en el proceso participaron 36 funcionarios administrativos y 32 representantes de los organismos de socorro.

Posteriormente, se contrastó la información obtenida con las perspectivas de cada uno de los actores, para lo cual se desarrollaron seis grupos de enfoque (uno por municipio), con el objetivo de reconocer las opiniones y significaciones construidas por lo actores de los entes territoriales y organismos de socorro en torno a los procesos de GRD definidos por la ley, su desarrollo y cumplimiento en los contextos de su injerencia. La discusión y negociación entre los participantes se cumplió a partir de los mismos elementos de los cuales se recabó información con el inventario. Los encuentros tuvieron una duración promedio de dos horas, fueron grabados en audio con autorización expresa de los participantes y se desarrollaron en sitios de trabajo facilitados por las propias administraciones locales.

\section{Análisis de la información}

Se cumplió en dos momentos, por un lado, la información obtenida con el inventario y las evidencias aportadas por los municipios fueron revisadas con fines de verificación y, a su vez, como estrategia de control que permitiera corroborar la veracidad de los resultados que se desprendieran de los grupos de enfoque, es decir, facilitó contrastar en qué medida las significaciones construidas por los actores correspondían con la realidad documentada. Con esto se realizó una descripción del material existente e inexistente en los entes territoriales frente a las evidencias solicitadas.

Por otra parte, la información obtenida en los encuentros de los grupos de discusión fue organizada mediante la técnica de sistematización propia de la IAP, y el análisis de sus contenidos se cumplió según los fundamentos de la Teoría Fundamentada (Grounded Theory), identificando contenidos o categorías semánticas centrales, las cuales se descompusieron en contenidos segmentarios asociados con los principales; finalmente, se realizó la depuración de la información mediante el criterio de redundancia teórica eliminando así la información repetida y resumiendo los contenidos en una única red de significaciones semánticas, la cual se construyó con ATLAS.ti 7.1.8@ para definir los tipos de relaciones (dependencia, causa, asociación, oposición) entre las categorías semánticas y los temas que las componían.

\section{RESULTADOS}

La aplicación del inventario para la recolección de evidencias documentales sobre procesos de GRD permitió identificar un panorama general del estado de los procesos en los diferentes municipios. Con el fin de ser concisos en la presentación de la información, en la Tabla 1 se describen los datos más relevantes que fueron identificados. 
Tabla 1. Resultados identificados en la revisión de evidencias documentales relacionadas con el desarrollo de procesos de GRD en los municipios estudiados
Cumplimiento de las disposiciones generales de la norma
- Ninguno de los municipios cuenta con personal experto en GRD vinculado al gabinete.
- No existe dedicación exclusiva para funciones de GRD ni creación de cargos en las administraciones con funciones basadas en dicho proceso.
- Las funciones de coordinaciónen procesos de GRD son asumidas regularmente por las direcciones de planeación.
- No se han definido planes de contingencias ajustados a las amenazas de cada territorio.
- No hay definidos procedimientos para el desarrollo y sostenimiento de sistemas de información y comunicación ante desastres.
- En ninguno de los seis municipios existe documentación sobre ocurrencia de eventos de desastres.
- No existen censos confiables ni definitivos de la población en riesgo de desastres. Información sobre el funcionamiento delCMGRD

- En los seis municipios los CMGRD fueron creados por decreto.

- La composición de los consejos se ajusta estrictamente a lo establecido literalmente en el artículo 28 de la Ley 1523 de 2012, incluyendo a veces, cuerpos o representantes que no existen en algunos municipios (p.e.: se define como parte del CMGRD al cuerpo bomberil, pero se carece del mismo).

- No se integra a miembros de la comunidad como parte del CMGRD.

- No existen planes de trabajo fijados en coordinación con los planes de desarrollo o con los planes de GRD.

- Las capacitaciones a los integrantes del Consejo han sido escasas y generadas desde iniciativas externas pero no por gestión de este organismo.

- No se han definido planes de contingencia.

Estado de las entidades técnicas y de socorro

- Reducido nivel de apropiación de los contenidos y alcances de norma por parte de organismos técnicos y de socorro.

- Baja coordinación en la comunicación de información pertinente entre entes administrativos y organismos de socorro (p.e.: algunos municipios cuentan con mapas de riesgos pero la Defensa Civil no los conoce pues el acceso es restringido).

- Bajo nivel de equipamiento de los organismos de socorro y ausencia de registros de gestión territorial para su dotación. 
Manejo financiero de GRD

- Creación del fondo para el manejo de GRD dentro de los términos y plazos definidos por la ley.

- Declaración de las respectivas subcuentas de Conocimiento, Atención y Mitigación.

- Cuentas sin recursos o con un nivel muy bajo de los mismos en relación con la magnitud de las amenazas.

- Se ha incluido en algunos municipios la GRD como parte de la asignación presupuestal, pero los recursos disponibles son significativamente bajos.

Participación sociocomunitaria

- No hay integración oficial de líderes de las comunidades en el CMGRD.

- Escaso número de actividades de socialización y formación.

- No hay registros (posteriores a la Ley 1523) de participación en ejercicios de simulacros.

- No se registran actividades relacionadas con educación en construcción social del riesgo.

- El sistema de atención a las comunidades se basa en el soporte humanitario de emergencia.

Fuente: los sutores.

En resumidas cuentas, un número importante de acciones definidas por la ley no han sido desarrolladas en los entes territoriales, y en otras ocasiones los procesos de tipo legal y normativo como la constitución de los CMGRD y las cuentas para tal gestión han tenido algunas dificultades. Esta realidad será ampliada en las discusiones generadas en los grupos de enfoque.

Al respecto, vale decir que los análisis de los grupos de discusión establecidos en cada municipio generaron un alto nivel de redundancia teórica, lo que parece sugerir cierto nivel de acuerdo en materia de las concepciones que socialmente se entretejen frente al tema en discusión por parte de los representantes de las administraciones locales y los organismos de socorro. En total se obtuvieron 15 códigos semánticos derivados de los análisis descritos los cuales abarcaron una temática amplia, desde el concepto general de la ley, hasta las concepciones sobre el desempeño institucional. La red semántica finalmente depurada aparece en la Figura 1. 


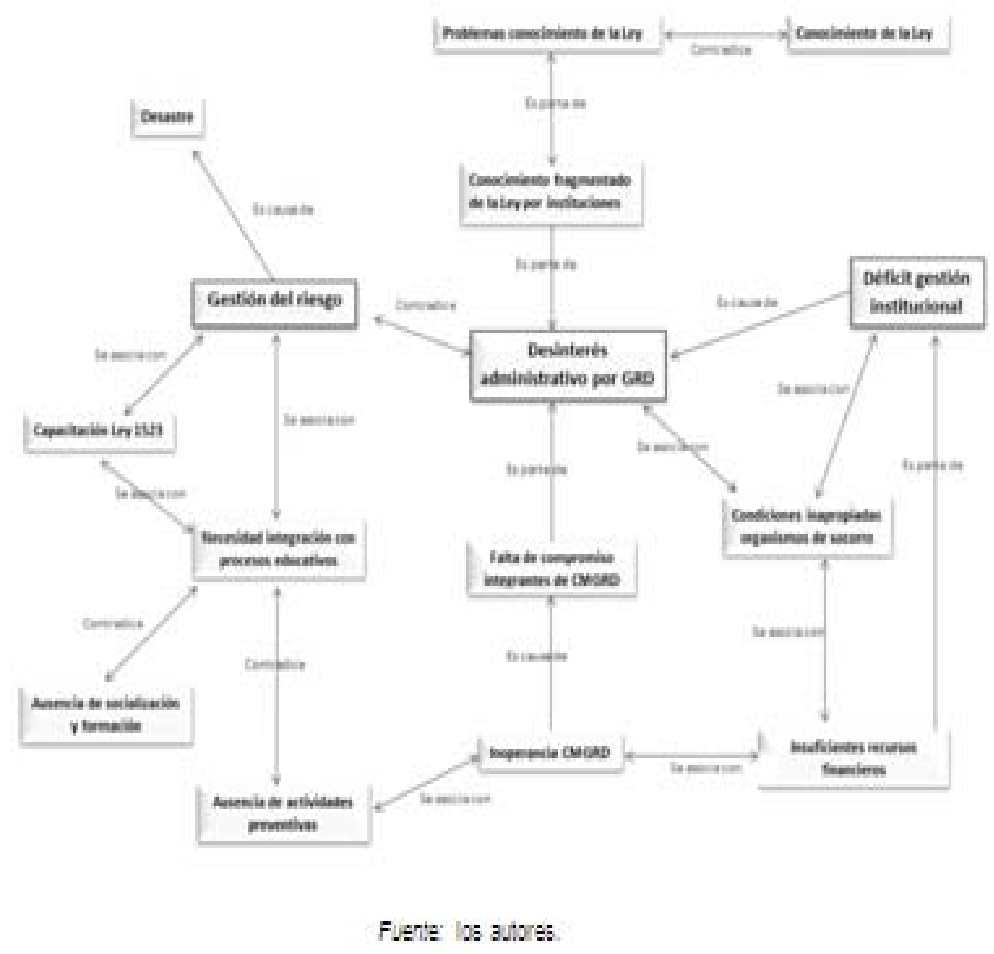

Figura 1. Red semántica organismos de socorro y las administraciones locales.

Haga clic sobre la imagen para ampliarla

Los enfoques fueron diversos y las posiciones pluralistas, pero en general sobresalió la riqueza semántica de temáticas como Gestión del riesgo, Desinterés administrativo por la GRD y Déficit de gestión institucional. A continuación se describirán estos resultados.

\section{Gestión del riesgo}

Este proceso, sobre el cual se centra toda la lógica de política pública que busca educar (generar conocimiento) y prevenir los desastres, aparece como un proceso derivado de la calamidad, es decir, se reseña una lógica contraria al espíritu de la ley. Esto puede estar ligado a la experiencia del contexto frente a la experimentación de las inundaciones y la posterior aparición de la Ley 1523, sin embargo, tal evidencia denota lo perdurable de ciertas ideas que siguen asociando el trabajo sobre los desastres con su atención más que con su gestión, p.e.: "[a] nosotros lo que nos preocupa es una solo cosa y es la prevención y atención de desastres $^{6}[\ldots] "$ " (Líder Defensa Civil, municipio de Suan).

Esta connotación denota reactividad frente al desastre, lo cual es precisamente una de las inadecuadas prácticas que busca cambiar la ley, sin embargo, no puede negarse que existe entre los grupos de trabajo concepciones favorables frente a la existencia de una ley para la GRD, la cual se asume como una normatividad vigente y de obligatorio cumplimiento y señalan la necesidad de integrarla a procesos educativos desde los cuales se contribuya a la capacitación sobre la Ley 1523.

En este tópico hay al menos dos posturas, por un lado, los participantes señalan que la comunicación y socialización de la norma ha sido muy reducida, y por el otro, precisamente hacen hincapié en la necesidad de generar estrategias educativas que integren la comunicación por parte de la institucionalidad territorial, 
con la participación de los núcleos de formación a nivel escolar en procesos de GRD. En este sentido, los mismos actores reconocen un problema en materia de difusión de la GRD y plantean una posible y accesible solución ante lo que consideran es una ausencia de socialización y ausencia de actividades preventivas.

\section{Desinterés administrativo por la GRD}

Propuestas como la de integrar la formación educativa con procesos de gestión del riesgo pueden ser viables para la difusión y generación de conciencia sobre dichos procesos, sin embargo, estas iniciativas han encontrado talanqueras que se atribuyen al poco acompañamiento institucional para dar vuelo a la gestión del riesgo.

Los integrantes de las administraciones locales y organismos de socorro definen como una especie de desinterés administrativo la falta de cooperación que en sus municipios ofrecen las instituciones, evidenciada en un desconocimiento de los pormenores relacionados con la Ley 1523 y sus disposiciones, la ausencia de mecanismos para el cumplimiento de la misma, entre otros, aunque esta no es una responsabilidad que se atribuye exclusivamente al representante legal de los municipios sino que involucra a otros actores que pueden incluso hacer caso omiso a las disposiciones municipales, en los casos en que los alcaldes intentan fomentar la GRD como se los reseña a los alcaldes de Suan y Repelón. Un caso muy llamativo lo representan los CMGRD, señalados por la falta de compromiso de sus integrantes, veamos pues, algunos testimonios:

[...] bueno yo se los diría así, cuesta reunirlos si ustedes se dan cuenta, aquí no hay representantes de la Policía, no hay representantes de la ESE, siempre brillan por su ausencia [pausa reflexiva] ¡Educación!... se pueden dar cuenta quienes estamos acá [...]. (Representante Defensa Civil, municipio de Suan)

[...] vemos que cada vez que se necesita una reunión del Consejo, siempre somos como los mismos y las personas que representan a las demás secretarías no se acercan. (Integrante CMGRD, municipio de Repelón)

Aquí se hacen las reuniones cuando amenaza un evento [...] cuando se hacen convocatorias es muy poca la asistencia [...] eso sí, siempre nos ha pasado y no solo en esta administración también en anteriores, entonces qué es lo que pasa, que uno convoca y siempre vienen los mismos [...] entonces no es porque no queramos reunirnos es por lo que te digo: la participación, la participación. (Secretario de Planeación, municipio de Santa Lucía)

Las ausencias continuas y sin justificación a las citaciones, la falta de planes de trabajo organizados y acordes a la realidad de los municipios e incluso el desconocimiento acerca de los procesos de GRD por parte de los integrantes del CMGRD han conducido a estas percepciones que acarrean además la noción de dicho Consejo como inoperante, lo cual se ve agravado por el reconocimiento de una tendencia a ofrecer respuestas reactivas ante las situaciones de amenaza, p.e.:

[...] si no unificamos criterios vamos a andar mal y siempre todo va a andar mal, como pasa siempre y como pasó en el incendio ${ }^{?}[\ldots]$ ha pasado la tragedia, lo que podemos llamar tragedia ecológica [...] pero no nos hemos reunido y siempre pasa lo mismo, de pronto nos van a reunir 
después de que pasa la tragedia, pero antes para tratar de prevenir no, no... no se hace, entonces siempre vamos a estar así tomando las mismas medidas. (Integrante Defensa Civil, municipio de Repelón)

Según estos resultados, el reducido nivel de participación de quienes integran el CMGRD deriva en su inoperancia, siendo a su vez, un segundo factor que podría explicar la falta de actividades preventivas, la educación y la socialización que previamente se discutía. Los testimonios señalan que la figura del CMGRD aparece como un requisito legal para el cumplimiento de las disposiciones definidas en la Ley 1523, pero el trabajo basado en la planificación de una agenda coherente con el plan de desarrollo y con el plan de gestión del riesgo y la estrategia de respuesta a la emergencia es deficiente. Sin duda, la falta de un organismo que regule, promueva y supervise mediante seguimientos detallados la educación y prevención en los temas atinentes al escenario de los riesgos, reduce toda posibilidad de generar estrategias de comunicación social capaces de permear al grueso de la población y, más aun, de producir sentido de apropiación por la cultura preventiva.

A todo lo anterior se suma el reconocimiento de niveles bajos de manejo de los contenidos y alcances de la Ley 1523; se señala incluso que la misma se ha manejado de forma fragmentada y que cada institución se centra en los puntos de su propio interés, mientras que la lógica sistémica y de integralidad que la ley contempla en sus principios es así ignorada.

\section{Déficit de gestión institucional}

Aunque en la red semántica construida, el déficit de gestión institucional aparece como producto del reducido interés administrativo por la GRD, merece un análisis particular por los elementos que comporta. Inicialmente sobresale el reconocimiento de uno de los principales tropiezos para la GRD en los municipios estudiados, la falta de recursos económicos dispuestos para este fin.

En el contexto colombiano este resultado parece una obviedad, dado que los municipios del sur de Atlántico son entes territoriales de sexta categoría, es decir, cuentan con un número inferior a 10 mil habitantes y un ingreso anual promedio inferior a 15 mil salarios mínimos colombianos (una suma cercana a los 5 millones de dólares), según lo establece el artículo 6 de la Ley 617 de 2000 (Ministerio de Hacienda y Crédito Público, 2000).

En este sentido, gran parte de las dificultades asociadas con el desarrollo de los procesos de GRD en los municipios estudiados son atribuidas a la existencia de insuficientes recursos financieros, esta realidad, parece ser bastión de defensa justificadora incluso para los rezagos de orden cultural y administrativo frente al tema. Sin embargo, el trasfondo del fenómeno supera las limitaciones económicas en lo meramente relacionado con los ingresos territoriales, pues incluye también el cometido institucional en materia de búsqueda de soluciones a partir de la gestión a nivel público y privado, la cual precisamente, hasta la fecha ha sido bastante reducida.

El trabajo de campo desarrollado en este estudio permitió corroborar que efectivamente no existen reportes oficiales de gestiones nacionales 0 internacionales dirigidas a la generación de recursos que permitan intervenciones dentro del marco de las exigencias de la ley, salvo aquellas acciones emprendidas en materia de corrección, arreglo o control de cuerpos de agua, las cuales han sido financiados a través del Fondo Nacional de Gestión del Riesgo de Desastres según el reporte de julio de 2014 de la subcuenta Colombia Humanitaria, con un monto superior a los 45 mil millones de pesos colombianos. 
La gestión con otras instituciones ha sido muy poca, el desarrollo de estrategias para unirse entre municipios que comparten riesgos ha sido mínimo e improductivo, la gestión de ayuda internacional es prácticamente nula, y en el peor de los casos, algunas administraciones expresaron en los grupos de trabajo que no se presentan propuestas a organismos internacionales cooperantes para dichos temas puesto que carecen de personal con la formación y el conocimiento para el diseño y presentación de proyectos, o simplemente no hay dolientes que asuman tales responsabilidades.

Finalmente, la noción de déficit institucional también atañe al papel que desempeñan los organismos de socorro, los cuales son vistos como indispensables pues son los primeros que se presentan ante los desastres e incluso quienes con mayor voluntad se han apropiado de la prevención, pero las condiciones estructurales son muy desfavorables dado que en la mayoría de los casos, Defensa Civil por ejemplo, carece de elementos materiales mínimos para el trabajo, no cuenta con sistemas de información ni con dotación, e incluso, no tiene sedes. Un caso muy llamativo fue hallado en uno de los municipios, donde la sede de la Defensa Civil se encuentra en una zona con alto riesgo de inundación que en el pasado ha sufrido pérdida de las pocas herramientas y elementos materiales con los que contaban, producto del desbordamiento del arroyo que bordea la casa donde se improvisa dicha sede. Tales condiciones han conducido a que incluso los miembros de organismos de socorro no hayan acudido a las últimas emergencias generadas por incendios forestales, dado que al carecer de elementos mínimos de protección, sus líderes han optado por preferir la seguridad e integridad del personal antes que por atender la emergencia.

\section{DISCUSIÓN}

El desarrollo de este estudio se enfocó en el análisis de la forma como se ha desarrollado la implementación de la política de GRD según las disposiciones de la Ley 1523 de 2012 en una jurisdicción territorial con serios antecedentes de experimentación de desastres derivados de eventos naturales (sur de Atlántico), para ello, el análisis se centró sustancialmente en un enfoque social, desde el cual, la percepción y la construcción de significaciones de la institucionalidad y de los organismos de socorro se ha considerado como un termómetro de mucho valor para conocer las posibles falencias y los obstáculos que han tenido lugar en la implementación de dichos procesos.

A través de este estudio se ha dirigido la atención a la "voz" de los actores institucionales y sociales, entendiendo que el contenido social se ciñe a las representaciones de los conglomerados objeto de estudio sobre los desastres y a los contenidos de lenguaje que representan, construyen y configuran la realidad social. Los desastres tienen un contenido subjetivo puesto que la forma como los grupos le interpretan es disímil, y de igual forma, esta naturaleza comprensiva es altamente significativa en el estudio de los procesos de gestión frente al riesgo, pues involucran directamente a las personas y a quienes les representan en un marco democrático participativo en el cual, además, están insertos los procesos de GRD.

Los resultados obtenidos demarcan un horizonte de complicaciones y limitaciones en el cumplimiento de los procesos de GRD a nivel de las administraciones territoriales. En resumen, se puede señalar que la GRD como proceso de planeación y desarrollo social y comunitario se encuentra actualmente presa de circunstancias irregulares asociadas con la gestión pública, las cuales tienden a 
desvirtuar el objeto de la política producto de la forma como se aplica en lo territorial. En el colectivo estudiado sobresalen lecturas que demarcan la poca funcionabilidad institucional observada en la falta de cumplimiento de los procesos de GRD, la ausencia de mecanismos propicios de planificación frente al riesgo, y más aún, la reducida acción de los CMGRD, con los consecuentes reparos sobre su compromiso e involucramiento en esta importante tarea de la gestión pública. Asimismo, los resultados muestran cómo la gestión es asumida como una tarea llena de limitaciones, lo cual se aprecia en los bajos niveles de asociatividad y generación de cooperación con otros organismos de orden nacional e internacional, la ausencia de recursos económicos suficientes y la falta de dotación de insumos necesarios para que los organismos de socorro logren desarrollar su labor y actuación ante los desastres que se puedan presentar en su jurisdicción.

Amén de lo anterior, el panorama advierte sobre la manifiesta necesidad de vincular de una forma más participativa a la comunidad en el proceso, en especial porque muchos grupos comunitarios viven en medio de un riesgo palmario, pero aun así su injerencia en la política requiere de mayor impacto; estas combinaciones (déficit gestión institucional - déficit participación comunitaria) constituyen una limitante significativa sobre el cumplimiento a nivel territorial de los procesos de GRD. Así pues, en el contexto específico de estudio el horizonte que se muestra no es para nada alentador, dado que muy a pesar de la normatividad vigente, el sistema de aplicación resulta inapropiado lo que limita a la territorialidad en la posibilidad de paliar los inconvenientes en cuanto al conocimiento, prevención y reducción del riesgo.

Este tipo de resultados señalan cierto nivel de divorcio entre los ideales normativos inspirados en la administración sustentable del territorio mediante la inclusión de la GRD, con las acciones específicas de planeación y desarrollo local, lo que sin duda pone al descubierto las debilidades experimentadas en el plano regional y la exposición a riesgos en la que permanecen las comunidades. La carencia de adecuados procesos de articulación entre la política propuesta a nivel nacional y su aplicación en el contexto urbano-rural afecta considerablemente el nivel de autonomía con el que se ejecutan los procesos de gestión pública, especialmente porque dejan en entredicho la posición de la institucionalidad limitando la relevancia de los instrumentos normativos (Narváez et al., 2009).

Este tipo de situaciones desdibuja los alcances de la gestión pública, la cual acude precisamente a la GRD en la medida en que esta última le surte de una serie de beneficios que redundan en el cumplimiento de adecuados procesos de administración territorial (Lavell, 2006; Velásquez, 2011), sin embargo, para que la gestión del riesgo cumpla este cometido se requiere su aplicación con un sentido transversal, en una práctica organizada desde lo institucional (Lavell, 2003), elementos que al parecer se adeudan en el contexto abordado en este estudio.

Coincidimos con Lampis (2010a) en que el riesgo -y el manejo inadecuado del mismo que deriva en el desastre- constituye un serio elemento que afecta las capacidades de los grupos sociales y mengua su posibilidad de desarrollo, por lo cual es indispensable elevar el nivel de efectividad de las instituciones públicas y de los organismos de la sociedad civil en la aplicación de mejores estrategias de desarrollo que coadyuven a la planeación local enfocada en la gestión del riesgo, para superar así las limitaciones al desarrollo sociocomunitario mediante la reducción de la vulnerabilidad social.

En un trasfondo más profundo, la superación de las debilidades a las que se expone la sociedad producto del manejo inadecuado del riesgo implica una reforma sustancial en la gobernanza del mismo, la cual debe alimentarse de un 
mayor nivel de compromiso político con el fin de gestar articulaciones legítimas con el nivel de gobierno nacional, así como con otros actores en el escenario local que expresen competencia y voluntad política, esto incluye también que la gobernanza en cuestión se apropie de las necesidades y de los potenciales aportes de la sociedad civil (Gellert de Pinto, 2012).

La experiencia adquirida a nivel internacional debe servir de ejemplo para el logro de este nivel de aplicación y legitimidad de la política de gestión del riesgo, en la medida en que los resultados obtenidos en otras latitudes refuerzan las ideas previamente expresadas. Llosa y Zodrow (2011), exponen en los resultados de su estudio sobre la aplicación de normatividad para la reducción del riesgo en países de altos y medianos ingresos, que a pesar del incremento de los esfuerzos en la creación de políticas sectoriales y de instrumentos a nivel jurídico para la gestión del riesgo, sigue existiendo una brecha considerable entre la acción emprendida en el orden nacional y la aplicación en las esferas locales. Entonces, no resulta suficiente contar con un aparato normativo robusto frente a la GRD sino que el éxito de la misma recae sobre la posibilidad de apropiación en el plano local y en la aplicación de mecanismos de gobernanza política que, como describe Gellert de Pinto (2012), constituyan una reforma desde la concepción misma del riesgo para pasar a la gestión integral pensada en el desarrollo del territorio.

Lo anterior, obliga a considerar las previsiones que deben ser asumidas en la aplicación de políticas públicas de GRD con un sistema descentralizado como ocurre en Colombia, puesto que incluso en naciones con una estructura administrativa y legislativa sólida que aplican políticas de gestión descentralizadas, se observa un distanciamiento a nivel aplicado, es decir, la estructura de la normatividad pierde coordinación y coherencia al momento de ser aplicada en los distintos sectores, lo cual pone en peligro la eficacia en materia de reducción del riesgo (Holub y Fuchs, 2009).

Tal y como señalan Scott y Tarazona (2011), los beneficios de la descentralización en las políticas de GRD a menudo se asocian con la búsqueda de un mayor nivel de participación por parte de los diferentes actores locales en la política nacional, al otorgar más autonomía en lo sectorial y local se busca una mayor integración con la toma de decisiones y a su vez se fomenta la cultura de la revisión, la autogestión económica y la rendición de cuentas. Sin embargo, este horizonte sufre tropiezos en la práctica, uno de los más comunes radica en la falta de recursos generados por el gobierno local, lo que conduce a que la financiación de la GRD continúe en gran medida centralizada, así mismo, los acuerdos y las voluntades políticas son comúnmente una talanquera a estos procesos conllevando a la falta de capacidades administrativas producto del poco interés por parte de los políticos locales para el cumplimiento de los compromisos públicos (Scott y Tarazona, 2011).

Esto exige que la aplicación de políticas públicas de esta naturaleza y envergadura coexista con procesos de reforma del aparato institucional, al cual se le demanda una ampliación de los estudios en materia de mecanismos para el correcto desarrollo de la política pero también el fortalecimiento técnico a nivel regional de las instituciones encargadas de la protección civil, y la ejecución de procesos de coordinación que alineen el servicio de las instituciones públicas con los intereses y necesidades sociales (Linayo, 2012). Al mismo tiempo, resulta necesario que los actores sociocomunitarios no sean invitados invisibles en los procesos de planeación local, tanto por omisión de su participación por parte de los líderes gubernamentales, como por la propia falta de involucramiento por parte de los actores comunitarios y de los organismos de protección que les representan, pues es a través de la conformación de veedurías ciudadanas que se 
logra un adecuado proceso de seguimiento y verificación del cumplimiento de la política.

El control de los alcances y la aplicación de la política pública de GRD no se restringe exclusivamente a los actores implicados en su formulación, sino que abarca una diáspora más amplia en la que los miembros de la sociedad civil y sus instituciones cobran un rol protagónico. En sus significaciones, su interpretación de la realidad y la vivencia misma frente al riesgo, hay un cúmulo de experiencias que enriquecen la planeación local del riesgo de desastre, desconocerle es limitar los alcances analíticos frente al tema y disgregarle de la realidad contextual de las comunidades.

Los resultados de este este estudio descuellan construcciones sociales que conjugan la representación colectiva del quehacer de la política con la concepción institucional del mismo, tales construcciones son relevantes en cuanto definen la forma como la gestión territorial es percibida, al igual que su legitimidad y la coherencia que se aprecia entre la administración de orden nacional y aquella que se ejecuta en el contexto específico de desarrollo de las comunidades, así pues, este tipo de análisis deja entrever la importancia de los procesos sociales involucrados en la formación de conceptos (Douglas, 1996) sobre el riesgo y sobre su gestión.

Reconocer la riqueza de los elementos sociológicos y políticos implicados en la GRD es también un aspecto valioso en los procesos encaminados a su estudio, de hecho, a manera de cierre, podemos señalar que el advenimiento de las ciencias sociales y de las ciencias humanas en el estudio de las amenazas y los desastres marca un cambio de paradigma en las concepciones sobre la intervención pública y retorna la cuestión central a su cauce principal, es decir le reconoce como un asunto sociopolítico, porque el problema público de los desastres tiene una alta implicación sociopolítica. Dado que los aspectos técnicos del estudio del riesgo muestran un elevado nivel de aplicación de las ciencias exactas con métodos maduros y probados, su utilización solo está limitada por la disponibilidad presupuestal que tienen las regiones, de manera que el problema técnico termina siendo de naturaleza política, es decir sobre la decisión de utilizar los recursos para ello o para otra labor.

Desde un enfoque constructivista del estudio de las políticas públicas, los conceptos de desastre, amenaza, vulnerabilidad, riesgo y gestión del riesgo asumen una connotación propia de las ciencias políticas, la sociología y la psicología social, donde lo público, las representaciones sociales y la construcción social de la realidad a través del lenguaje, toman relevancia explicativa.

\section{CONCLUSIONES}

La comprensión conceptual de la gestión del riesgo en Colombia apenas está siendo asimilada en pequeños círculos de los estudiosos del tema, que producto del proceso experimental de situaciones trágicas ante la ocurrencia de desastres, han asumido el análisis y la discusión, no solo de los impactos que estos eventos producen, sino también de las causas y el proceso mediante el cual se construye la vulnerabilidad al riesgo de ocurrencia de los mismos. Por otro lado, la institucionalidad encargada de dirigir la gestión de este proceso (entidades públicas territoriales y nacionales, empresas privadas y sociedad civil organizada), pese a estar sujeta a la imposición de la norma que hoy lo regula, desconoce su contenido conceptual y desestima los mandatos que de ella emanan. 
Es de reconocerse el acierto del gobierno nacional en la formulación de un política de GRD y su consecuente adopción mediante la Ley 1523 de 2012, a la cual se le reconoce su pertinencia, pero en cuanto a su correcta aplicación, resultan notorias las debilidades en la capacidad institucional de las entidades territoriales para gestionar el proceso de implementación, debilidades soportadas inicialmente en el desconocimiento de los alances de la norma y de las herramientas que esta aporta para su implementación, y de manera estructural, en la escasa capacidad institucional para ejecutar las decisiones emanadas de la norma.

Estas limitaciones en la capacidad institucional se evidencian principalmente en la ausencia de funcionalidad de los CMGRD como instancia orientadora y rectora de la ejecución de los procesos de conocimiento y mitigación del riesgo, y preparación para el manejo de desastres, y de ahí en adelante, la inexistencia de procesos institucionales que promuevan la apropiación del conocimiento sobre el tema, de acuerdo con el énfasis que hace la norma para su implementación. Y es que el protagonismo de la institucionalidad en el posicionamiento de cualquier política pública es de absoluta relevancia, pues las instituciones tienen, en principio, una capacidad de incidencia en la sociedad, pero esa capacidad para producir cambios está condicionada -en una relación de recíproca incidencia- por la existencia de estructuras profundas de tipo cultural, político o económico (García et al., 2011).

Pero estas estructuras son susceptibles de ser transformadas en la medida en que se implementen procesos formativos y educativos asociados con acciones que despierten el interés común, como lo son el bienestar y la seguridad territorial. La gestión del riesgo entonces, requiere de un vasto proceso de socialización colectiva sobre su conceptualización, su historia y la dimensión de su aplicación, para que las capacidades institucionales de las entidades públicas se complementen y se fortalezcan con la dinámica activa y propositiva de la academia, los gremios privados y los procesos sociales y comunitarios.

A partir de esta realidad, es imperativo que las instituciones del orden nacional (Unidad Nacional de Gestión del Riesgo de Desastres, ministerios e institutos descentralizados), que por razones lógicas tienen una mayor comprensión de lo que implica la apropiación de la política pública de GRD en los niveles territoriales y en la ciudadanía en general, asuman el apoyo técnico financiero para el desarrollo de este proceso de socialización colectiva, de forma coordinada y articulada con estas instancias, de tal manera que se eviten los esfuerzos aislados que hoy atentan con los principios y objeto de la norma, y que son percibidos por las administraciones territoriales y los ciudadanos como acciones transitorias.

\section{AGRADECIMIENTOS}

Los autores reconocen y agradecen la contribución ofrecida en el marco del proyecto al cual se adscribe esta producción, a los profesionales Oswaldo Rapalino Carroll (REDePARES), Eduardo Polo, Liliana Anaya, Vilma Solano (Asociación de Egresados de la ESAP - regional Costa Caribe), Nicolás Salinas, Ailed Marenco, Adriano Portillo, Rosa Hernández (Corporación Universitaria Reformada) y Natalia Sáenz Rengifo (Instituto de Estudios del Ministerio Público). 


\section{CONFLICTO DE INTERESES}

Los autores declaran que no hay conflicto de intereses.

\section{FUENTE DE FINANCIACIÓN}

Este artículo se desprende del trabajo de campo desarrollado en el marco del proyecto de investigación: "Análisis de la política de gestión del riesgo en comunidades del Caribe colombiano afectadas por desastres invernales: el caso del sur del departamento de Atlántico" (Código 180061138309), financiado mediante convocatoria pública por el Departamento Administrativo de Ciencia, Tecnología e Innovación (Colciencias) de Colombia.

\section{REFERENCIAS}

- Audefroy, J. (2007). Desastres y cultura: Una aproximación teórica. Revista Invi, 60(22), 119-132.

- Balcázar, P., González, N., Gurrola, G. \& Moysén, A. (2005). Investigación cualitativa. México: UNAM.

- Banco Interamericano de Desarrollo -BID- y Comisión Económica para América Latina y el Caribe -Cepal-. (2012). Valoración de daños y pérdidas. Ola invernal en Colombia 2010-2011. Bogotá: BID.

- Banco Mundial. (2012). Análisis de la gestión del riesgo de desastres en Colombia: Un aporte para la construcción de políticas públicas. Bogotá: Banco Internacional de Reconstrucción y Fomento - Banco Mundial Región de América Latina y El Caribe.

- Beck, U. (1998). La sociedad del riesgo. Barcelona: Paidós.

- Cardona, O. D. (1993). Evaluación de la amenaza, la vulnerabildiad y el riesgo. En Marskrey, A. (Comp.), Los desastres no son naturales (pp. 5174). Bogotá: La Red.

- Colombia Humanitaria. (2014). Fondo Nacional de Gestión de Riesgo de Desastres. Subcuenta Colombia Humanitaria. Balance Atlántico. Informe a $\begin{array}{llll}\text { corte Julio } 14 & \text { de } 2014 . & \text { Recuperado }\end{array}$ de http://www.colombiahumanitaria.gov.co/FNC/Documents/2011/padrinos /atlantico.pdf

- Congreso de la República. (2012). Ley 1523. Diario Oficial, 48411). Bogotá.

- Douglas, M. (1996). La aceptabilidad del riesgo según las ciencias sociales. Barcelona: Paidós.

- Dwyer, A., Zoppou, C., Nielsen, O., Day, S. y Roberts, S. (2004). Quantifying social vulnerability: a methodology for identifying those at risk to natural hazards. Canberra: Geoscience Australia.

- Fundación Red para la Promoción, Prevención, Atención y Recuperación de Emergencias -REDePARES-. (2013). Documento técnico. Informe de municipios del sur de Atlántico. Barranquilla.

- García, M., García, M., Rodríguez, J. C., Revelo, J. y Espinosa, J. (2011). Los Estados del país. Instituciones municipales y realidades locales. Bogotá: Centro de Estudios de Derecho, Justicia y Sociedad, DeJuSticia. 
- García, V. (2005). El riesgo como construcción social y la construcción social de riesgos. Revista Desacatos, 19, 11-24.

- Gellert de Pinto, G. (2012). Latín A: El cambio de paradigma: de la atención de desastres a la gestión del riesgo. Boletín Científico Sapiens Research, 2(1), 13-17.

- Herzer, H. M. (2011). Construcción del riesgo, desastre y gestión ambiental urbana: Perspectivas en debate. Revista Virtual Redesma, 5(2), 51-60.

- Holub, M. y Fuchs, S. (2009). Mitigating mountain hazards in Austria legislation, risk transfer, and awareness building. Natural Hazards and Earth System Sciences, 9, 523-537.

- Keipi, K., Mora, S. y Bastidas, P. (2005). Gestión de riesgo de amenazas naturales en proyectos de desarrollo. Lista de preguntas de verificación ("Checklist"). Serie de informes de buenas prácticas del Departamento de Desarrollo Sostenible. Washington, D.C.: Banco Interamericano de Desarrollo.

- Lampis, A. (2010a). ¿Qué ha pasado con la vulnerabilidad social en Colombia? Conectar libertades instrumentales y fundamentales. Sociedad y Economía, 19, 229-261.

- Lampis, A. (2010b). Pobreza y riesgo medioambiental: Un problema de Vulnerabilidad y Desarrollo. Working Paper. Centro Interdisciplinario de Estudios sobre Desarrollo. Bogotá: Universidad de los Andes.

- Lavell, A. (2000). Sobre la Gestión del Riesgo: Apuntes hacía una Definición. Recuperado de http://www.bvsde.paho.org/bvsacd/cd29/riesgoapuntes.pdf

- Lavell, A. (2003). La Gestión Local del Riesgo. Nociones y precisiones en torno al concepto y la práctica. Guatemala: CEPREDENAC - PNUD.

- Lavell, A. (2006). Del concepto de riesgo y su gestión a los parámetros para la acción: un resumen básico. PREDECAN.

- Lavell, A. (2007). Apuntes para una reflexión institucional en países de la Subregión Andina sobre el enfoque de la Gestión del Riesgo. Apoyo a la Prevención de Desastres en la Comunidad Andina -PREDECAN-. Recuperado

de http://www.comunidadandina.org/predecan/doc/r1/docAllan2.pdf

- Linayo, A. (2012). Damnificados nuestros de cada día. Revista Planeo. Instituto de Estudios Urbanos y Territoriales de la Pontificia Universidad Católica de Chile. Recuperado de http://www.desenredando.org/public/2012/2012_A_Linayo_planeo.ieut. cl_damnificados

- Llosa, S. y Zodrow, I. (2011). Disaster risk reduction legislation as a basis for effective adaptation. In Global Assessment Report on Disaster Risk Reduction. United Nation Office for Disaster Risk Reduction (UNISDR). Recuperado de http://www.preventionweb.net/english/hyogo/gar/2011/en/bgdocs/Llosa \&_Zodrow_2011.pdf

- Luján, J. L. y Echeverría, J. (2009). Gobernar los riesgos. Ciencia y valores en la sociedad del riesgo. Madrid: Organización de Estados Iberoamericanos -OEI-.

- Martí, J. (2000). La investigación acción participativa. Estructura y fases. En: T. Villasante., M. Montañez. \& J. Martí. La investigación social participativa. Construyendo ciudadanía I (pp. 73-117). Madrid: El viejo Topo.

- Ministerio de Hacienda y Crédito Público. (2000). Ley 617. Diario Oficial, 44.188. Bogotá.

- Morales-Soto, N., Gálvez-Rivero, W., Chang-Ausejo, C., Alfaro-Basso, D., García-Villafuerte, A., Ramírez-Maguiña, M. et al. (2008). Emergencias y 
desastres: Desafíos y oportunidades (De la casualidad a la causalidad). Revista Peruana de Medicina Experimental y Salud Pública, 25(2), 237-242.

- Narváez, L., Lavell, A. y Pérez, G. (2009). La gestión del riesgo de desastres: un enfoque basado en procesos. San Isidro, Perú: Secretaría General de la Comunidad Andina.

- PNUD. (2013). Curso de gestión de riesgos y gobiernabildiad local. Unidad I: Conceptualización del desarrollo humano y gestión integral del riesgo. PNUD. Escuela virtual del PNUD.

- Reveiz, E. (2007). El Estado regulador de riesgos. Bogotá: Academia Colombiana de Ciencias Económicas -ACCE-.

- Sánchez, A. (2011). Después de la inundación. Documento de trabajo sobre economía regional. Cartagena: Banco de la República.

- Scott, Z. y Tarazona, M. (2011). Study on Disaster Risk Reduction, Decentralization and Political Economy. Decentralisation and Disaster Risk Reduction. In Global Assessment Report on Disaster Risk Reduction. United Nation Office for Disaster Risk Reduction (UNISDR). Recuperado de http://www.preventionweb.net/english/hyogo/gar/2011/en/bgdocs/Scott_ \&_Tarazona_2011.pdf

- Thomas, J. E. (2011). Desarrollo y gestión social del riesgo: ¿Una contradicción histórica? Revista de Geografía Norte Grande, 48, 133-157.

- Velásquez, L. S. (2011). La gestión del riesgo en el contexto ambiental urbano local un reto permanente y compartido. Caso Manizales Colombia. Medio Ambiente y Urbanización, 75(1), 27-46.

- Wilches-Chaux, G. (1993). La Vulnerabilidad Global. En Marskrey, A. (Comp.), Los desastres no son naturales (pp. 11-44). Bogotá: La Red.

1. Magíster en Psicología. Corporación Universitaria Reformada. Barranquilla, Colombia.javila@unireformada.edu.co

2. Magíster en Estudios Políticos. Instituto de Estudios del Ministerio Público. Bogotá, Colombia. oavivasc@procuraduria.gov.co

3. Magíster en Dirección Estratégica con énfasis en Gerencia. Escuela Superior de Administración Pública (ESAP). Barranquilla, Colombia. herreaf@gmail.com

4. Profesional en Administración Pública. Asociación de egresados de la ESAP (ASESAP) Costa Caribe. Barranquilla, Colombia. micaribejd@gmail.com

5. Esta idea de una transmutación del problema público se refleja en la documentación que marca la acción pública y en documentos internacionales como los del PNUD (PNUD, 2013).

6. El énfasis es nuestro.

7. Conflagración ocurrida en el mes de abril por acción antrópica; el incendio arrasó con varias hectáreas de bosque seco tropical incluidas fincas de pequeños lugartenientes y campesinos. 
Para citar este artículo: Ávila-Toscano, J. H., Vivas Cortés, O. A., Herrera Flórez, A. y Jiménez Díaz, M. (2016). Gestión del riesgo de desastres en el Caribe colombiano desde la óptica de organismos de socorro y administraciones locales: el caso del sur de Atlántico. Revista Luna Azul, 42, 68-88. Recuperado de http://vip.ucaldas.edu.co/lunazul/index.php?option=com_content\&view=article\&id= 129 